\title{
Role of Synaptic Inhibition in the Coupling of the Respiratory Rhythms that Underlie Eupnea and Sigh Behaviors
}

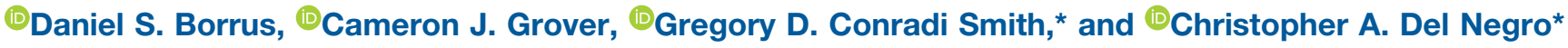

https://doi.org/10.1523/ENEURO.0302-19.2020

Department of Applied Science, Integrated Science Center, William \& Mary, Williamsburg, VA 23185

\begin{abstract}
The preBötzinger complex (preBötC) gives rise to two types of breathing behavior under normal physiological conditions: eupnea and sighing. Here, we examine the neural mechanisms that couple their underlying rhythms. We measured breathing in awake intact adult mice and recorded inspiratory rhythms from the preBötC in neonatal mouse brainstem slice preparations. We show previously undocumented variability in the temporal relationship between sigh breaths or bursts and their preceding eupneic breaths or inspiratory bursts. Investigating the synaptic mechanisms for this variability in vitro, we further show that pharmacological blockade of chloride-mediated synaptic inhibition strengthens inspiratory-to-sigh temporal coupling. These findings contrast with previous literature, which suggested glycinergic inhibition linked sigh bursts to their preceding inspiratory bursts with minimal time intervals. Furthermore, we verify that pharmacological disinhibition did not alter the duration of the prolonged interval that follows a sigh burst before resumption of the inspiratory rhythm. These results demonstrate that synaptic inhibition does not enhance coupling between sighs and preceding inspiratory events or contribute to post-sigh apneas. Instead, we conclude that excitatory synaptic mechanisms coordinate inspiratory (eupnea) and sigh rhythms.
\end{abstract}

Key words: breathing; central pattern generator; preBötzinger complex

\section{Significance Statement}

Normal breathing consists of eupnea and sigh breaths, which differ in their magnitude, frequency, and function. Both breath types emerge from a brainstem microcircuit that coordinates their timing. Here, we advance understanding of these rhythms by assessing the nature and strength of their coordination, and by showing that synaptic inhibition does not constrain their temporal coupling in contrast to conventional understanding. This study elucidates synaptic mechanisms linking oscillations of different amplitude and frequency within one core oscillator microcircuit.

\section{Introduction}

Under physiological conditions breathing behavior consists of two interleaving rhythms and motor patterns: eupnea and sighing. Eupnea is the normal unlabored breathing that underlies rhythmic lung ventilation and drives alveolar gas exchange. Eupnea occurs at $\sim 1-4 \mathrm{~Hz}$ in rodents $(0.2-0.3 \mathrm{~Hz}$ in humans); each breath ventilates a small fraction of lung capacity. Sighs are also inspiratory

Received August 1, 2019; accepted May 1, 2020; First published May 11, 2020.

The authors declare no competing financial interests. breaths, but the volume of inhaled air during a sigh is two to five times that of a normal breath, and sigh frequency is an order of magnitude lower than the eupnea rhythm ( $\mathrm{Li}$ and Yackle, 2017). Sighs reinflate collapsed (or collapsing) alveoli and are essential for optimal pulmonary function. Typically, sighs appear to ride atop ongoing eupneic breaths (Cherniack et al., 1981; Orem and Trotter, 1993),

Author contributions: D.S.B., G.D.C.S., and C.A.D.N. designed research; D.S.B., C.J.G., and C.A.D.N. performed research; D.S.B. and G.D.C.S. contributed unpublished reagents/analytic tools; D.S.B., C.J.G., and G.D.C.S. analyzed data; D.S.B., G.D.C.S., and C.A.D.N. wrote the paper. 
which suggests that periodically, but at a much lower frequency, the eupnea cycle triggers the sigh. After a sigh, the next eupneic breath is delayed for a duration roughly equivalent to one additional eupneic cycle (Cherniack et al., 1981; Orem and Trotter, 1993). This delay, which we refer to as the post-sigh apnea, suggests that sighs prolong the time to the next eupneic breath. Eupnea and sigh breathing rhythms thus appear to be coupled, most likely via neural microcircuits of the brainstem that generate and control breathing movements.

In mammals, eupnea and sigh rhythms emanate from the preBötzinger complex (preBötC) of the lower brainstem (Smith et al., 1991; Del Negro et al., 2018). Both rhythms are maintained in reduced slice preparations that isolate the preBötC as well as inspiratory premotor and motor neurons, and thus encapsulate a minimal breathing-related model system (Lieske et al., 2000; Ruangkittisakul et al., 2008; Chapuis et al., 2014). Because eupnea refers to behavior in living animals, inspiratory is the appropriate nomenclature for eupnearelated activity in slice preparations. Inspiratory rhythm depends on network properties, in which recurrent excitation among glutamatergic interneurons is rhythmogenic (Funk et al., 1993; Rekling et al., 2000; Del Negro et al., 2002; Wallén-Mackenzie et al., 2006; Feldman and Kam, 2015; Ashhad and Feldman, 2020). The rhythmogenic mechanism of sighs is unknown, but maintenance of the sigh rhythm depends on neuropeptides released by parafacial respiratory interneurons (Li et al., 2016) as well as excitatory ionotropic and metabotropic receptor-mediated synaptic transmission (Lieske and Ramirez, 2006a,b).

Inspiratory bursts appear to trigger sigh-related bursts, and in turn, sigh-related bursts delay the next inspiratory burst by almost an entire cycle (Lieske et al., 2000; Tryba et al., 2008). These observations in vitro mirror the in vivo coupling behavior described above, which suggest that the mechanisms coupling inspiratory and sigh rhythms are contained within the preBötC and can be examined at the cellular and synaptic level in vitro.

What mechanisms couple these two rhythms? The only existing data suggest that glycinergic synaptic inhibition links the sigh-related burst to its preceding inspiratory burst, thus giving rise to the biphasic shape in which the inspiratory burst appears to trigger the sigh (Lieske et al., 2000). A recent mathematical model (Toporikova et al., 2015) posits two discrete systems for generating eupnea and sigh oscillations. The model inspiratory system acts on the sigh system via synaptic inhibition such that sigh bursts emerge via an escape-like process triggered by

This work was supported by National Institutes of Health Grants R01HL104127 (to C.A.D.N.) and AT010816 (to G.D.C.S. and C.A.D.N.).

${ }^{*}$ G.D.C.S. and C.A.D.N. contributed equally to this work.

Correspondence should be addressed to Christopher A. Del Negro at cadeln@ wm.edu.

https://doi.org/10.1523/ENEURO.0302-19.2020

Copyright $(2020$ Borrus et al.

This is an open-access article distributed under the terms of the Creative Commons Attribution 4.0 International license, which permits unrestricted use, distribution and reproduction in any medium provided that the original work is properly attributed. disinhibition at the tail end of inspiratory bursts. The model also suggests that the sigh system projects to the inspiratory system via fast excitatory synapses, and the strength of its excitation leads to a transient state of refractoriness, i.e., the post-sigh apnea, in the coupled system. However, the post-sigh apnea might also be attributable to synaptic inhibition from the sigh system onto the inspiratory rhythm generator.

Here, we challenge two longstanding ideas regarding the sigh rhythm: that when a sigh occurs, it emerges immediately following the associated eupneic breath, and that synaptic inhibition is responsible for this temporal relationship. First, we describe previously undocumented variability in the timing of a sigh breath relative to the previous eupneic event. We show, both in vivo and in vitro, that a sigh event can occur simultaneously, and even precede, the inspiratory event. Next, we block glycinergic transmission and show that disinhibiting the preBötC in vitro does not uncouple the eupnea- and sigh-related rhythms, but in fact appears to couple them more strongly. We obtain similar results when simultaneously blocking GABAergic and glycinergic transmission.

We extend our investigation of synaptic inhibition in coupling the two breathing rhythms by analyzing the post-sigh apnea in vitro before and after blockade of ionotropic inhibition. We show the duration of the post-sigh apnea does not depend on glycinergic or GABAergic transmission and we infer that the post-sigh apnea reflects a refractory state attributable to postsynaptic membrane properties evoked by the sigh burst. Lastly, we measure the chloride reversal potential $\left(E_{C I}\right)$ in putatively rhythmogenic preBötC neurons and meta-analyze the development of $\mathrm{E}_{\mathrm{Cl}}$ in mice and rats, to verify that glycine and $\mathrm{GABA}_{A}$ synapses are inhibitory postnatally, and not functionally excitatory as they are during embryonic development (Ren and Greer, 2006; Delpy et al., 2008). We propose that the eupnea and sigh rhythms are coupled predominantly by excitatory (rather than inhibitory) synaptic mechanisms.

\section{Materials and Methods}

\section{Ethical approval and animal use}

The Institutional Animal Care and Use Committee at our institution approved these protocols, which conform to the policies of the Office of Laboratory Animal Welfare (National Institutes of Health) and the guidelines of the National Research Council [National Research Council (U.S.), 2011]. CD-1 mice (Charles River) and genetically modified mice (described below) were maintained on a $14 / 10 \mathrm{~h}$ light/dark cycle at $23^{\circ} \mathrm{C}$ and were fed ad libitum with free access to water.

To obtain patch-clamp recordings from preBötC neurons derived from progenitors that express the embryonic transcription factor Developing brain homeobox 1 (i.e., Dbx1), we used two Cre-driver mouse strains: homozygous knock-in mice generated by inserting an IRES-CREpGK-Hygro cassette in the $3^{\prime}$ untranslated region (UTR) of the $D b x 1$ gene, i.e., $D b x 1^{\text {Cre }}$ mice (Bielle et al., 2005; IMSR catalog \#EM:01924, RRID:IMSR_EM:01924) and 
homozygous knock-in mice generated by inserting an IRES-CreER ${ }^{\mathrm{T} 2}$ cassette in the $3^{\prime}$ UTR of the $D b x 1$ gene, which provides conditional Cre recombinase expression following activation of the tamoxifen-sensitive estrogen receptor, i.e., $D b \times 1^{\text {CreERT2 }}$ mice (Hirata et al., 2009; Picardo et al., 2013; IMSR catalog \#JAX:028131, RRID: IMSR_JAX:028131).

We crossed females of both strains with males from a reporter strain featuring Cre-dependent expression of the fluorescent $\mathrm{Ca}^{2+}$ indicator GCaMP6f dubbed Ai148 by the Allen Institute (Daigle et al., 2018; IMSR catalog \#JAX:030328, RRID:IMSR_JAX:030328). We refer to their offspring as Dbx1;Ai148 mice. During neonatal development and through adulthood, Dbx1;Ai148 mice express GCaMP6f in Dbx1-derived cells, the majority of which are neurons (Kottick et al., 2017).

\section{Breathing-related measurements in vitro}

Mouse pups of both sexes were anesthetized by hypothermia and then killed by thoracic transection at postnatal day $(\mathrm{P}) 0-\mathrm{P} 4$. The neuraxis was removed in $<2$ min and further dissected in artificial CSF (aCSF) containing the following: $124 \mathrm{~mm} \mathrm{NaCl}, 3 \mathrm{~mm} \mathrm{KCl}, 1.5$ $\mathrm{mm} \mathrm{CaCl}_{2}, 1 \mathrm{~mm} \mathrm{MgSO}_{4}, 25 \mathrm{~mm} \mathrm{NaHCO} 3,0.5 \mathrm{~mm}$ $\mathrm{NaH}_{2} \mathrm{PO}_{4}$, and $30 \mathrm{~mm}$ dextrose equilibrated with $95 \%$ $\mathrm{O}_{2}-5 \% \mathrm{CO}_{2}, \mathrm{pH} 7.4$. Isolated neuraxes were glued to an agar block and then cut in the transverse plane to obtain a single 550- $\mu \mathrm{m}$-thick slice that exposed the preBötC at its rostral face. Atlases for wild-type and $D b x 1$ reporter mice show that the loop of the inferior olive and the semicompact division of the nucleus ambiguus (NAsc) collocate with the preBötC during early postnatal development (Ruangkittisakul et al., 2011, 2014). Slices were then perfused with aCSF at $28^{\circ} \mathrm{C}$ in a recording chamber either below a stereomicroscope that enabled us to position suction electrodes under visual control, or on a fixed-stage microscope with dipping objectives and epifluorescence illumination to visually identify GCaMP6f-expressing (rhythmically active) Dbx1-derived neurons for patch-clamp recordings.

In both configurations, we elevated extracellular $\mathrm{K}^{+}$ concentration $\left(\left[\mathrm{K}^{+}\right]_{\mathrm{o}}\right)$ to $9 \mathrm{~mm}$ to increase preBötC excitability (Funk and Greer, 2013). Inspiratory-related motor output was recorded from the hypoglossal (XII) nerve rootlets, which are captured in transverse slices along with the XII motoneurons and their axon projections to the nerve rootlets, using suction electrodes and a differential amplifier. Field potentials were recorded from the preBötC by forming a seal over it with a suction electrode at the slice surface. Amplifier gain was set at 2000 and the bandpass filter was set at $300-1000 \mathrm{~Hz}$. XII and preBötC bursts were full wave rectified and smoothed for display and quantitative analyses of burst events.

We obtained on-cell patch-clamp recordings under visual control. Patch pipettes were fabricated from borosilicate glass (OD: $1.5 \mathrm{~mm}$, ID: $0.86 \mathrm{~mm}, 4-6 \mathrm{M} \Omega$ in bath) and filled with solution containing $150 \mathrm{~mm} \mathrm{KCl}$ and $10 \mathrm{~mm}$ HEPES). We added gramicidin (CAS number 1405-97-6, product G0550000 from Millipore Sigma) acutely at the start of the experiment from stock solution (2-mg gramicidin per 1-ml dimethyl sulfoxide) such that the final concentration was $20 \mu \mathrm{g} / \mathrm{ml}$. We also added Alexa Fluor 568 hydrazide (50 $\mu \mathrm{M}$, ThermoFisher Scientific) to the patch solution, which verifies the integrity of the gramicidin perforated patch as GCaMP6f fluorophore is expressed in the soma, whereas Alexa Fluor 568 is limited to the adjacent pipette. Patch pipettes with a bath resistance of 4-6 $\mathrm{M} \Omega$ were backfilled first with a drop of gramicidinfree patch solution to ensure gramicidin molecules did not interfere with the initial seal to the membrane. After backfilling, $200 \mu$ of patch solution with gramicidin was added to the pipette. We employed an EPC-10 patch-clamp amplifier exclusively in current-clamp mode (HEKA Instruments) because perforated patches cause a large access resistance at the interface between pipette and target neuron, which produces a voltage-divider circuit and precludes accurate voltage clamp.

We acquired and digitized the signals at $4 \mathrm{kHz}$ with a low-pass filter set to $1 \mathrm{kHz}$ using a 16-bit analog-to-digital converter (AD Instruments). All recordings were corrected offline for a liquid junction of $3.74 \mathrm{mV}$ (Barry and Lynch, 1991; Neher, 1992).

For both field recordings and associated perforatedpatch experiments, the glycine receptor antagonist strychnine hydrochloride (CAS number 1421-86-9, product S8753, Millipore Sigma) and the $\mathrm{GABA}_{\mathrm{A}}$ receptor antagonist picrotoxin (CAS number 124-87-8, product P1675, Millipore Sigma) were bath-applied at $5 \mu \mathrm{M}$.

All gramicidin perforated-patch experiments began no earlier than 30 min after achieving a seal on the plasma membrane exceeding $1 \mathrm{G} \Omega$ (i.e., gigaohm seal), which was sufficient for gramicidin to form ionophores and thus allow intracellular access and current-clamp recording. After we observed a stable recording configuration for $10 \mathrm{~min}$, which we considered the control conditions for subsequent analysis, then we applied strychnine and picrotoxin.

When locally applying $\mathrm{GABA}_{\mathrm{A}}$ and glycine receptor agonists (muscimol and glycine) onto patch-recorded neurons, we fabricated similar pipettes (as described above) from which to eject glycine and muscimol (dubbed "puffer" pipettes). Puffer pipettes were filled with $150 \mu \mathrm{m}$ glycine (CAS number 56-40-6, Millipore Sigma) and $30 \mu \mathrm{m}$ muscimol (CAS number 2763-96-4, Millipore Sigma) diluted into aCSF containing $9 \mathrm{~mm}\left[\mathrm{~K}^{+}\right]_{0}$ (as described above). Thirty minutes after forming a gigaohm seal on the plasma membrane, the puffer pipette was positioned to within $\sim 50 \mu \mathrm{m}$ of the neuron being recorded. Glycine and muscimol were ejected using 7-9 psi pressure pulses lasting $25-200 \mathrm{~ms}$, which we triggered by TTL commands from the EPC-10 amplifier.

Patch-recorded preBötC neurons were identified as neurons by their ability to discharge action potentials, recognizable $\sim 30 \mathrm{~min}$ after forming a gigaohm seal. The high impedance of gramicidin patches filters high frequency signals like action potentials, thus spike amplitude peaks below $-10 \mathrm{mV}$ while the integrity of the patch is maintained. The developmental genetic origin of neurons patched from wild-type preparations was not specified, thus we refer to these neurons as unspecified. Neurons 


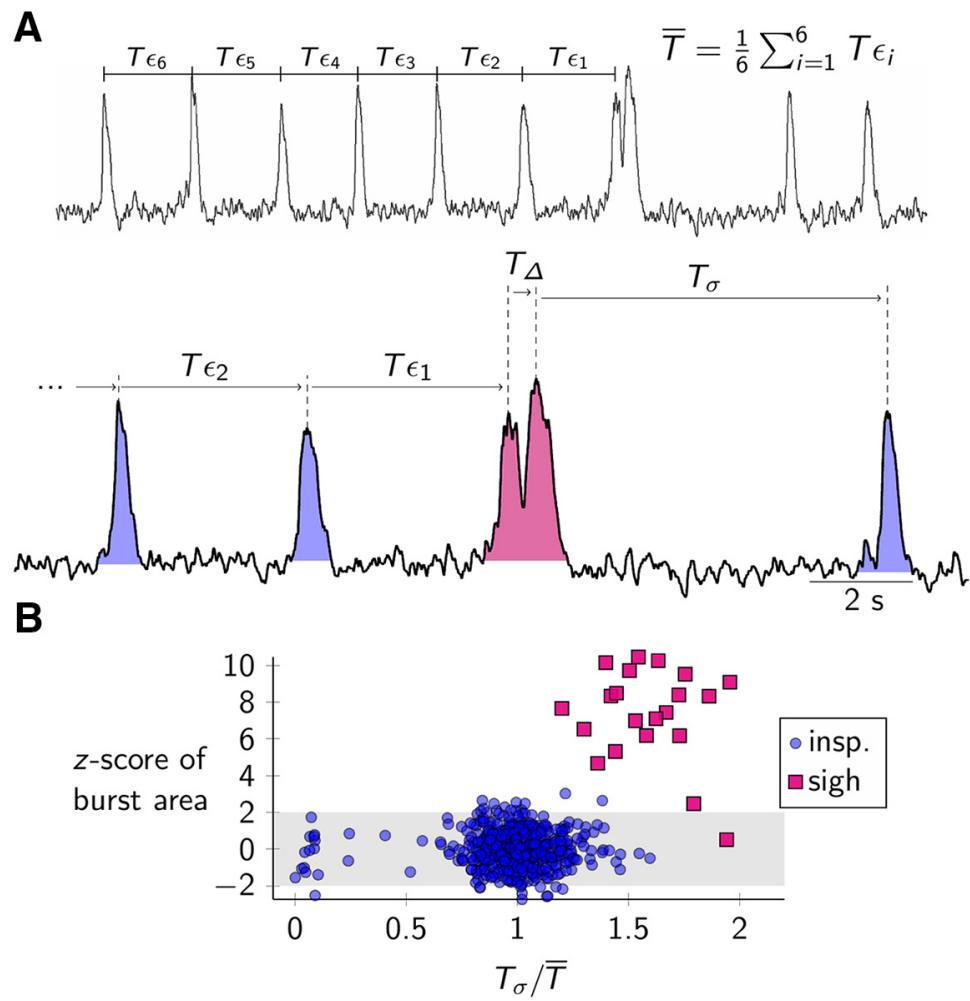

Figure 1. $\boldsymbol{A}$, Field recording of preBötC activity, which includes inspiratory and sigh bursts. Average inspiratory cycle time $(\bar{T})$ is calculated from six preceding cycle periods $\left(T_{\epsilon_{1}} \ldots T_{\epsilon_{6}}\right) . T_{\epsilon}$ represents period of a single inspiratory cycle; $T_{\Delta}$ represents the inspiratorysigh interval; $T_{\sigma}$ reflects the duration of the post-sigh apnea. $\boldsymbol{B}$, A scatter plot showing $z$ score transformation of burst area plotted against the post-sigh apnea duration, $T_{\sigma}$ (normalized to the average inspiratory cycle time, $\bar{T}$ ); $z$ score distribution is calculated from all bursts from this slice.

patched in Dbx1;Ai148 mouse slices, which were rhythmically active in sync with XII motor output, are referred to as Dbx1 neurons. For puffing experiments, we added 1 $\mu \mathrm{m}$ tetrodotoxin (TTX) to the bath after $>30 \mathrm{~min}$ to prevent spike-driven chemical synaptic transmission and isolate postsynaptic responses to muscimol and glycine. We held preBötC neurons at a range of baseline membrane potentials using bias currents. We measured membrane potential trajectories in response to puffed glycine and muscimol, in which the previous $2 \mathrm{~s}$ of membrane voltage were used as baseline.

\section{Breathing-related measurements in vivo}

We measured breathing in unrestrained, adult mice of both sexes ( $n=7$ mice total), using a whole-body plethysmograph (EMKA Technologies) with a balanced flow rate of $1 \mathrm{l} / \mathrm{min}$ in normoxia $\left(21 \% \mathrm{O}_{2}\right.$ and $\left.79 \% \mathrm{~N}_{2}\right)$. The mice were placed in the sealed chamber with constant air flow 10 min before data collection during each session for acclimation. We observed the mice during every session. Locomotion (exploring), grooming, and sniffing (with synchronized whisking) entrain and modify breathing so we only analyzed epochs of calm breathing absent orofacial or motor behaviors. A pneumotachograph with access to the chamber containing the mouse, and a reference chamber open to room air, were included in the circuit and connected to a differential pressure transducer
(AD Instruments, RRID:SCR_017551). The raw data reflect airflow, which was acquired and analyzed in LabChart 8 software (AD Instruments). Airflow was digitally integrated to determine volume changes during breathing and in particular to determine tidal volume $\left(\mathrm{V}_{\mathrm{T}}\right)$. The pressure transducer was calibrated by injecting 0.2 $\mathrm{ml}$ of air into the chamber during each trial. We analyzed continuous 60-min sessions of plethysmography. Epochs of calm breathing contain eupnea and sighs. Sighs were distinguished by inhaled volume exceeding $V_{T}$ by $2-3 \times$ (the inspired air during a sigh draws on the inspiratory reserve volume of the lungs, and thus exceeds $\mathrm{V}_{\mathrm{T}}$ by definition) and by the presence of a post-sigh apnea, i.e., a pause in breathing $1.3 \times$ longer than the average interbreath interval during eupnea.

\section{Identification and analysis of sigh bursts}

We measured burst area and cycle time for each event in preBötC field recordings (Fig. 1). We distinguished a sigh burst from an inspiratory burst by considering the area of an event in conjunction with the cycle time following that event. Sigh bursts have $z$ scores (mean/SD) for burst area that almost always exceed 2 (and are most often in the range 4-10), whereas inspiratory bursts have an average $z$ score of 0 (range -2-2). Sigh bursts are followed by a cycle time as long or longer than $1.3 \times$ the average cycle time for all events lumped together. Figure 
$1 A, B$ illustrates these measurements for a typical field recording.

The average inspiratory cycle time $(\bar{T})$ is computed from the six inspiratory cycles preceding the sigh $\left(T_{\epsilon_{1}} \ldots T_{\epsilon_{6}}\right) . T_{\Delta}$ is the inspiratory-sigh interval computed as the peak time of the sigh burst minus the peak time of the inspiratory burst. $T_{\sigma}$ is the duration of the post-sigh apnea. We normalize $T_{\Delta}$ and $T_{\sigma}$ by $\bar{T}$ to minimize the slice-by-slice variation in frequencies.

\section{Measurements and statistics}

We analyzed data and computed statistics using LabChart 7 \& 8 (AD Instruments), MATLAB 2018b (MathWorks, RRID: SCR_001622), and Igor Pro 8 (Wavemetrics, RRID:SCR 000325). We describe the statistical hypothesis tests used as they appear in the main text (Table 1).

\section{Results}

\section{Sigh breaths follow eupneic breaths with variable time intervals}

We recorded breathing in unanesthetized adult mice, analyzing 229 sigh breaths across seven animals. We measured the time interval between a sigh and its associated (generally preceding) eupneic breath, which we refer to as the eupnea-sigh interval. Figure 2 shows the distribution of the eupnea-sigh intervals with three representative traces corresponding to eupnea-sigh intervals that fall within three characteristic parts of the distribution. In 174 of the 229 sighs (76\%), the sigh emerged after the eupneic breath, leading to a positive eupnea-sigh interval (Fig. 2, trace a). This represents the canonical sigh pattern, whereby the inspired volume of the sigh builds off, and exceeds, the tidal volume marking the peak of a eupneic breath. Surprisingly however, the remaining 55 sighs $(24 \%)$ did not follow the canonical pattern. In 37 cases $(16 \%)$, the sigh appeared unaccompanied by a eupneic breath. We infer that the eupneic and sigh event occurred simultaneously, or close enough together so as to be indistinguishable, thus leading to an observed event interval of $0 \mathrm{~s}$ (Fig. 2, trace b). In 18 cases (8\%), the sigh breath was followed by a eupneic breath whose peak corresponds to tidal volume, then followed by a post-sigh apnea. This type of sigh pattern led to a negative eupneasigh interval (Fig. 2, trace c). The presence of null and negative eupnea-sigh intervals (Fig. 2, traces b and c, respectively) suggest that eupneic breaths are not obligatory for triggering a sigh.

\section{Sigh bursts follow inspiratory bursts with variable time intervals}

We next investigated the coupling relationship between eupnea and sigh rhythms using slice preparations that retain the preBötC and spontaneously generate inspiratory and sigh-related activity measurable in the preBötC and XII nerve rootlets. We measured 343 sigh bursts in 13 slice preparations ( $26 \pm 7$ sigh bursts per slice) via a field recording electrode. We measured the time interval between a sigh burst and its associated inspiratory burst, which we refer to as the inspiratory-sigh interval (Fig. 3A).
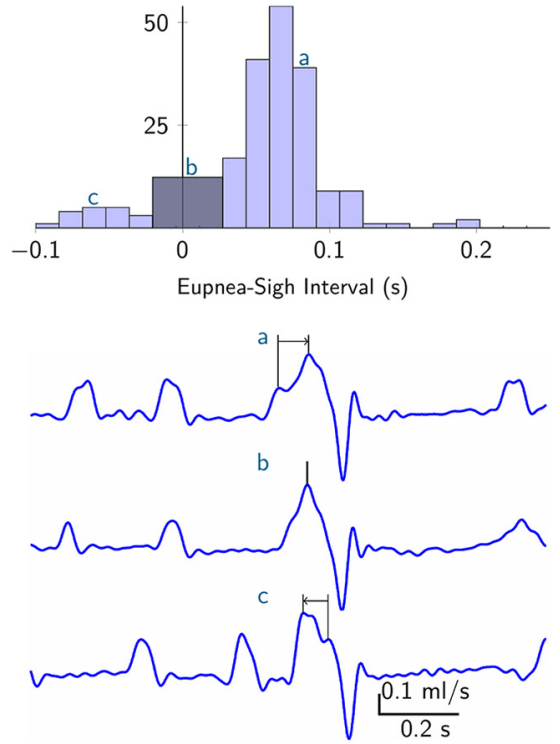

Figure 2. Variable timing of sigh breaths in vivo. Top, Histogram of the eupnea-sigh time intervals. Bin size is $0.016 \mathrm{~s}$. Intervals near zero were pooled into one bin of size $0.048 \mathrm{~s}$ because distinct eupnea-related and sigh-related peaks in the signal could not be distinguished. Bottom, Sample sweeps from plethysmography recordings from one mouse. Lower case letters $a, b$, and $c$ illustrate eupnea-sigh intervals that appear in corresponding bins plotted in the histogram above.

To account for the intrinsic slice-to-slice variation in inspiratory frequency, we normalized inspiratory-sigh interval $\left(T_{\Delta}\right)$ by the average of the six preceding inspiratory cycle periods $(\bar{T})$. Figure $3 A$ shows the distribution of inspiratorysigh intervals (top), as well as representative field recordings of sigh bursts with different inspiratory-sigh intervals that map to specific parts of the distribution (Fig. $3 A$ shows the correspondence via lower case letters a-e).

Inspiratory and sigh events recorded from XII motor output closely resemble the integrated preBötC field recording when considering inspiratory-sigh timing. We analyze the integrated field recording exclusively because it reflects activity of the core rhythmogenic microcircuits in the preBötC.

Inspiratory bursts tend to precede sigh bursts, as the distribution of inspiratory-sigh intervals is weighted toward positive values of $T_{\Delta} / \bar{T}$ close to 0 (Fig. $3 A$; e.g., trace $b)$. However, inspiratory-sigh intervals varied considerably. For example, only 133 sighs (39\%) appeared within the first fifth of the inspiratory cycle. Rather, we observed inspiratory-sigh intervals that ranged across the entire inspiratory cycle (Fig. $3 A$, traces b-e). Additionally, we observed 40 sigh bursts (12\%) that appeared to be followed by inspiratory bursts and then post-sigh apneas, thus indicating a negative inspiratory-sigh interval (Fig. $3 A$, trace a).

\section{Inhibitory synapses do not link sigh bursts to the inspiratory rhythm}

To perturb the temporal coupling between the inspiratory and sigh rhythms we blocked glycinergic synaptic 
A

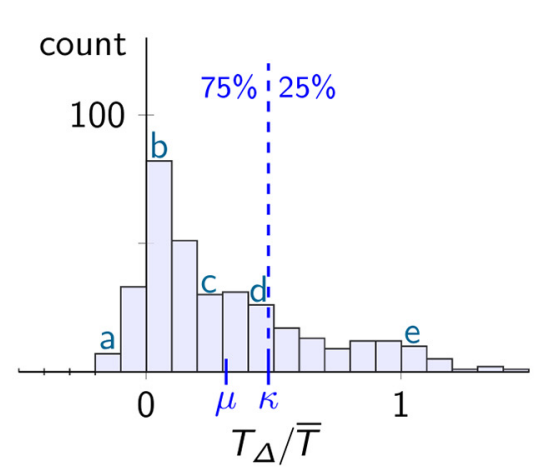

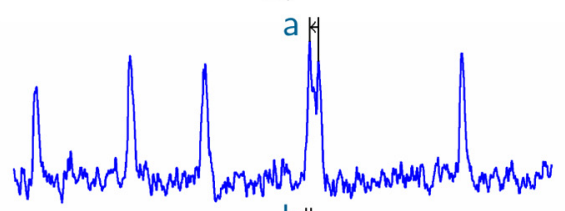
by

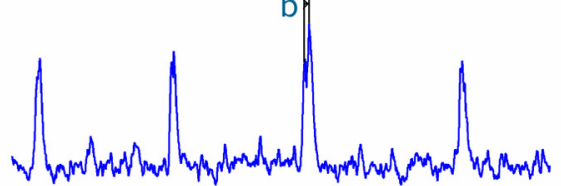

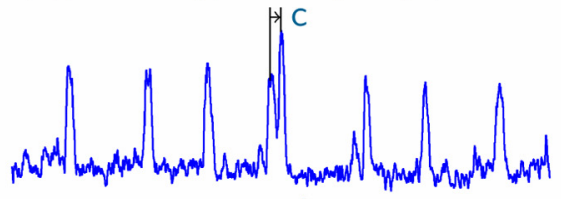<smiles>CCCCN1CCCC1NC(C)C</smiles>

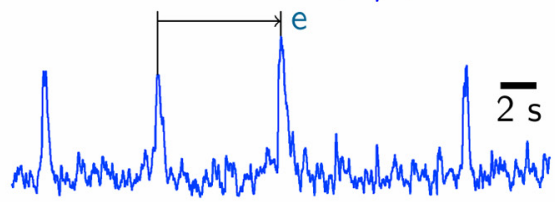

B

Strychnine
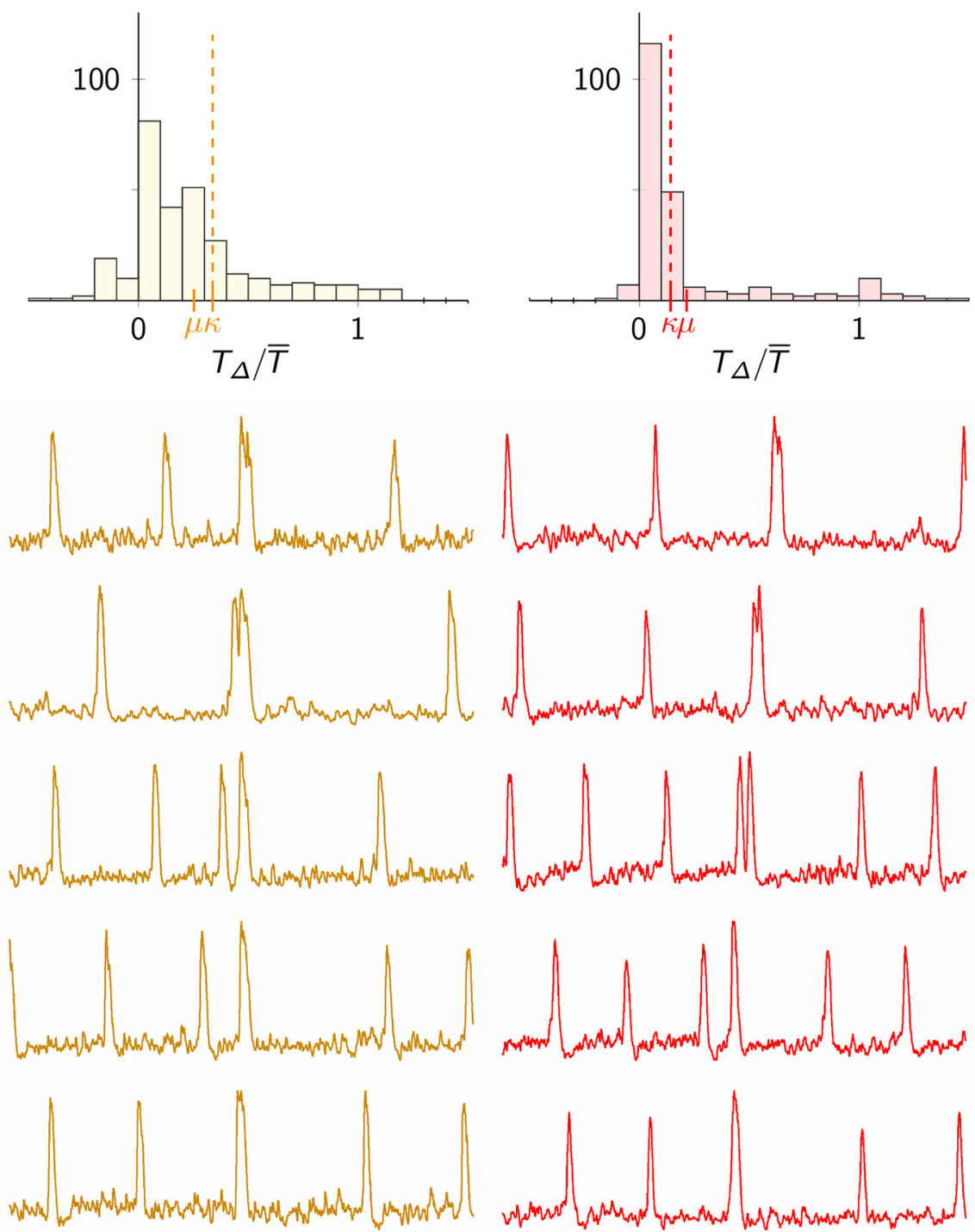

C Picrotoxin \& Strychnine

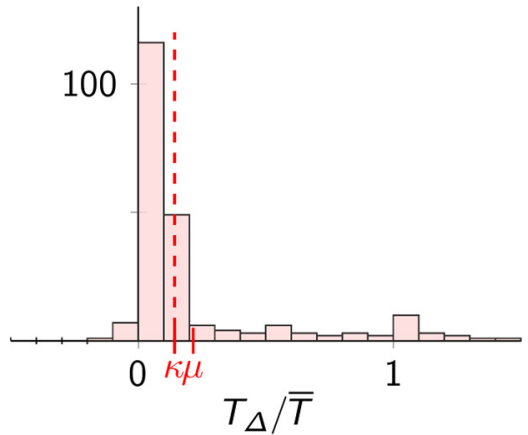

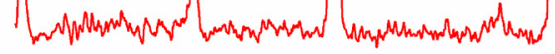

Figure 3. Variable timing of sigh bursts in vitro. $\boldsymbol{A}$, Histogram of normalized inspiratory-sigh intervals $\left(T_{\Delta} / \bar{T}\right)$ for all sigh bursts in all 13 rhythmically active slices under control conditions. The width of each bar is $0.1 T_{\Delta} / \bar{T} ; \mu$ marks the mean of the distribution, and $\kappa$ denotes the 75th percentile line. Below are representative preBötC field recording traces depicting a variety of inspiratory-sigh intervals. Traces come from different slices. Lower case letters a, b, c, d, and e connect representative traces with their position on the inspiratory-sigh interval histogram above. $\boldsymbol{B}, \boldsymbol{C}$, Identical inspiratory-sigh interval analysis in the presence of $5 \mu \mathrm{m}$ strychnine $(\boldsymbol{B})$ as well as both $5 \mu \mathrm{M}$ strychnine and $5 \mu \mathrm{m}$ picrotoxin $(\boldsymbol{C})$.

transmission using strychnine. The probability distribution of inspiratory-sigh intervals remained weighted toward the first half of the normalized cycle $\left(T_{\Delta} / \bar{T}<0.5\right.$; Fig. $\left.3 B\right)$, suggesting that inspiratory-sigh coupling remained intact. In contrast, previous studies suggested that blocking glycinergic synapses eliminated the temporal relationship between sigh bursts and their preceding inspiratory bursts (Lieske et al., 2000; Chapuis et al., 2014), in which case the probability distribution in Figure $3 B$ would be uniformly distributed between 0 and 1 . Here, the probability of short inspiratory-sigh intervals $\left(T_{\Delta} / \bar{T}<0.5\right)$ increased such that the mean inspiratory-sigh interval decreased from $T_{\Delta} / \bar{T}=0.31 \pm 0.34$ in control (Fig. $3 A, \mu$ ) to $T_{\Delta} / \bar{T}=$
$0.25 \pm 0.30$ in the presence of strychnine (Fig. $3 B, \mu)$. This trend is reflected in the significant leftward shift of the cumulative distribution function toward, but not below, an inspiratory-sigh interval of 0 from control to strychnine conditions (Kolmogorov-Smirnov, test statistic $=0.13$, $p=0.011, n=7$ slices; Fig. 4). The left shift of the entire inspiratory-sigh interval distribution is further illustrated by the decrease of the 75th percentile score (Figs. 3, 4, $\kappa$ ) from $T_{\Delta} / \bar{T}=0.48$ in control to $T_{\Delta} / \bar{T}=0.34$ in strychnine.

Similarly, when we simultaneously blocked glycine and $\mathrm{GABA}_{\mathrm{A}}$ receptors with a strychnine and picrotoxin cocktail, the sigh burst coupled more tightly with the preceding inspiratory burst than in control conditions, as shown by 


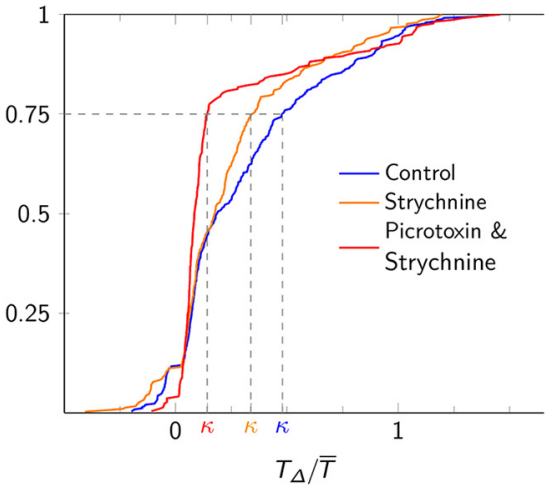

Figure 4. Cumulative probability histograms showing the normalized inspiratory-sigh interval $\left(T_{\Delta} / \bar{T}\right)$ under control conditions (blue), $5 \mu \mathrm{m}$ strychnine (orange), as well as $5 \mu \mathrm{m}$ strychnine and $5 \mu \mathrm{m}$ picrotoxin (red); $\kappa$ marks the 75th percentile line for each distribution, distinguished by color. This analysis pools all sighs measured across all 13 slices.

the accumulation of inspiratory-sigh intervals within the first two bins of the inspiratory-sigh interval histogram (Fig. 3C). The probability of short inspiratory-sigh intervals $\left(T_{\Delta} / \bar{T}<0.5\right)$ increased from control to the strychnine and picrotoxin cocktail such that the average normalized inspiratory-sigh interval decreased from $T_{\Delta} / \bar{T}=0.31 \pm 0.34$ in control (Fig. $3 A, \mu$ ) to $T_{\Delta} / \bar{T}=0.21 \pm 0.32$ in strychnine and picrotoxin (Fig. $3 C, \mu$ ). The significant leftward shift of the cumulative distribution function toward, but not below, an inspiratory-sigh interval of 0 further demonstrates that when both glycine and $\mathrm{GABA}_{\mathrm{A}}$ receptors were blocked, sigh bursts occurred earlier with respect to preceding inspiratory bursts than during control (KolmogorovSmirnov, test statistic $=0.31, p=4.0 \mathrm{E}-12, n=6$ slices; Fig. 4). The 75th percentile score (Figs. 3, 4, $\kappa$ ) drops from an inspiratory-sigh interval of $T_{\Delta} / \bar{T}=0.48$ in control to $T_{\Delta} / \bar{T}=$ 0.14 in strychnine and picrotoxin. Whereas in control and strychnine conditions $\mu<\kappa$ (the mean of the distribution was within the $0-75$ th percentile range) in the combined presence of strychnine and picrotoxin $\kappa<\mu$, which shows that the vast majority of inspiratory-sigh intervals (in 0-75th percentile range) are shorter than the mean.

Note that the large standard deviation associated with the average inspiratory-sigh intervals $(0.31 \pm 0.34$ in control, $0.25 \pm 0.30$ in strychnine, and $0.21 \pm 0.32$ in strychnine and picrotoxin) reflects the inherent inspiratory-sigh interval variability as illustrated in Figure 3.

Our analyses (Figs. 3,4 ) show the removal of chloridemediated synaptic inhibition does not uncouple the sigh from its preceding inspiratory burst, but rather disinhibition strengthened the temporal coupling of inspiratory and sigh bursts.

\section{Inhibitory synapses do not influence post-sigh apnea}

We calculated the relative duration of the post-sigh apnea as the duration of the post-sigh interval $\left(T_{\sigma}\right)$ divided by the average of the six preceding inspiratory cycle times $(\bar{T})$. We compared the relative duration of the post-sigh apnea $\left(T_{\Delta} / \bar{T}\right)$ in control and after blocking either

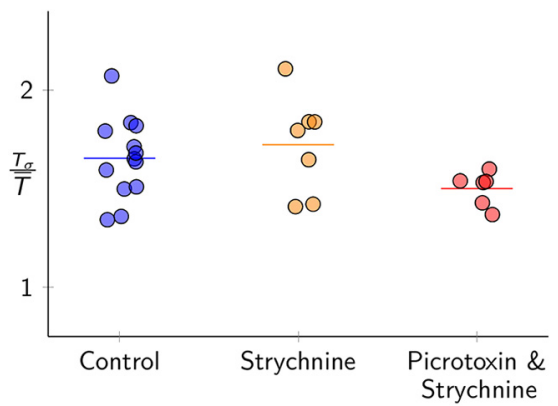

Figure 5. Average relative duration of the post-sigh apnea in control (blue), $5 \mu \mathrm{m}$ strychnine (orange), as well as $5 \mu \mathrm{m}$ strychnine and $5 \mu \mathrm{m}$ picrotoxin (red). Each dot represents the average normalized post-sigh apnea for all sighs recorded in one slice. Horizontal bars represent the average of all slices in each condition.

glycinergic transmission, or both glycinergic and ionotropic GABAergic transmission (Fig. 5). The relative duration of the post-sigh apnea measured $1.65 \pm 0.20$ in control, $1.72 \pm 0.25$ in strychnine, and $1.5 \pm 0.09$ in the strychnine-picrotoxin cocktail. These measurements are statistically indistinguishable (one-way ANOVA, $F_{(2,11)}=$ 2.12, $p=0.14, n=13$ slices).

\section{Chloride-mediated synaptic transmission is inhibitory in the neonatal mouse preBötC}

We recorded chloride-mediated synaptic potentials in preBötC neurons via gramicidin perforated patches during early postnatal development (P0-P3). These experiments employed wild-type mouse slices as well as slices from Dbx1;Ai148 mice that express a fluorescent $\mathrm{Ca}^{2+}$ reporter (GCaMP6f) in preBötC neurons derived from $D b x 1$ expressing progenitors (i.e., Dbx1 neurons), which comprise the core excitatory interneurons of the preBötC (Bouvier et al., 2010; Gray et al., 2010; Wang et al., 2014; Cui et al., 2016; Vann et al., 2016, 2018; Baertsch et al., 2018). Figure $6 A, B$ shows the neuroanatomical structures from a Dbx1;Ai148 slice that collocate with the preBötC: the principal loop of the inferior olive $\left(\mathrm{IO}_{\text {loop }}\right)$ and the NAsc. Figure $6 \mathrm{C}$ depicts the perforated patch configuration wherein GCaMP6f expression verifies that the neuron was $D b x 1$ derived. Figure $6 C$ also shows that Alexa Fluor 568 remains confined to the pipette solution during perforated patch recording conditions.

We applied strychnine and picrotoxin to the bath simultaneously while recording Dbx 1 inspiratory neurons $(n=4$ neurons in 4 slices). We monitored the integrated area of the membrane potential trajectory during inspiratory bursts while vigilantly monitoring the integrity of the perforated patch throughout the experiment using the absence of Alexa Fluor 568 in the soma as evidence of an intact patch (Fig. $6 C, D$ ). After blockade of chloride-mediated synapses, the average integrated area of inspiratory bursts increased from $2.2 \pm 0.7 \mathrm{~V} \times \mathrm{ms}$ in control to $5.2 \pm 2.1 \mathrm{~V} \times \mathrm{ms}$ (paired $t$ test, $t=3.6, p=0.037$; Fig. 6F). The significant increase in burst area suggests that chloride-mediated synapses are functionally inhibitory during network bursts. As a final check, we switched from a 

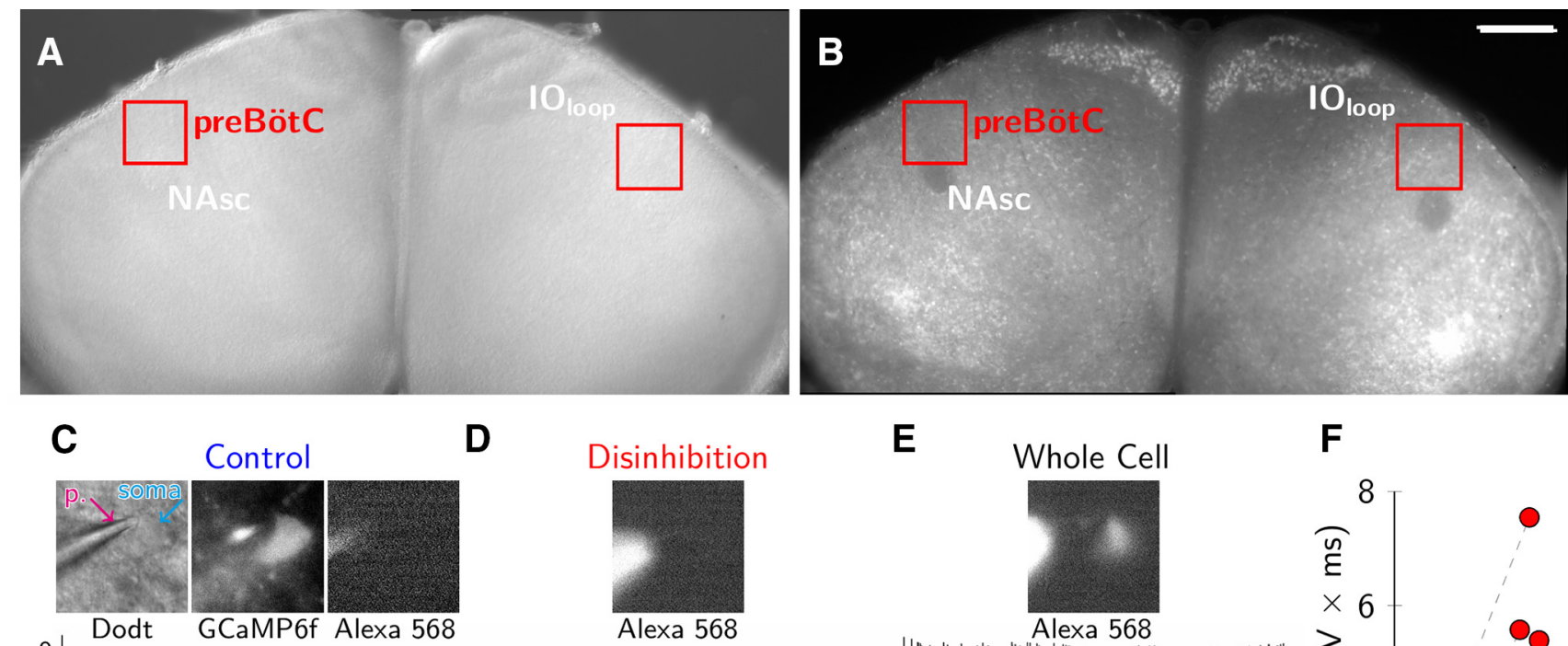

D

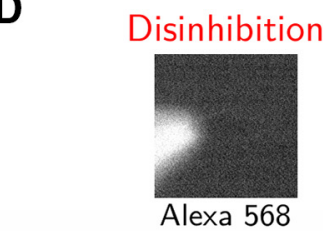

E
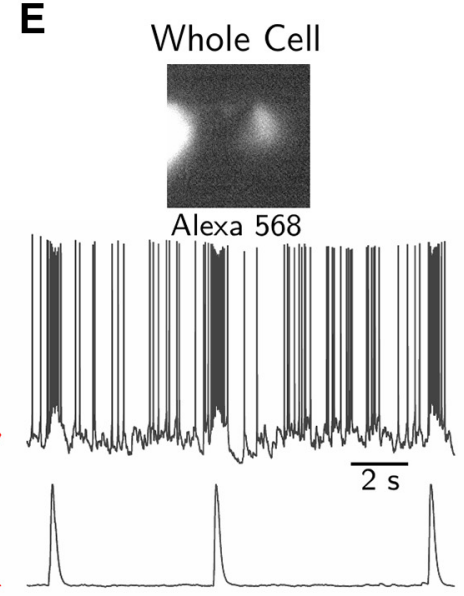

$\mathbf{F}$

$-60$
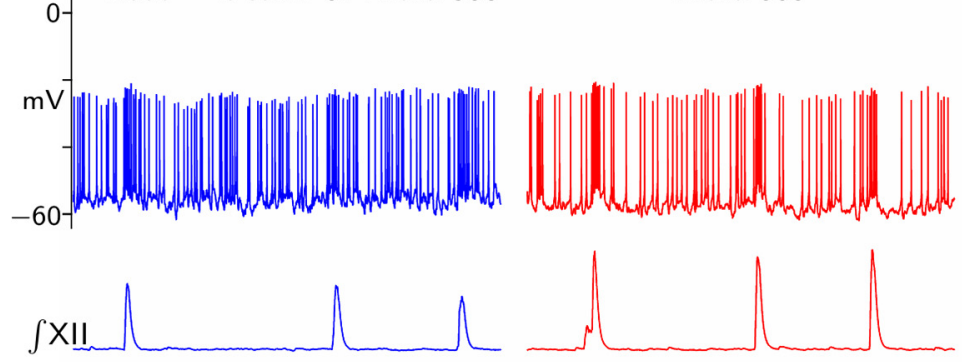

2
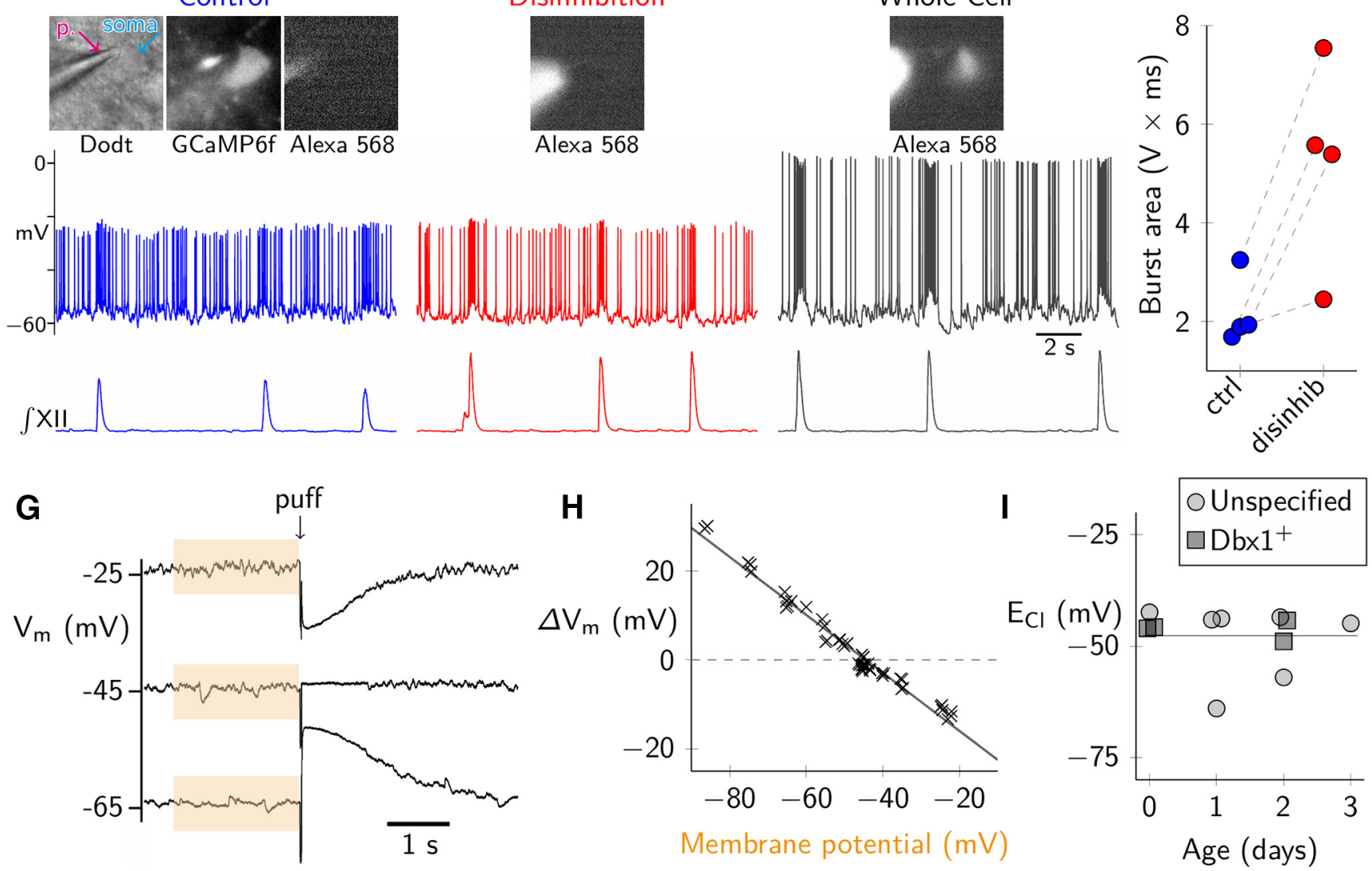

Figure 6. Chloride-mediated synapses are inhibitory in neonatal preBötC neurons. $\boldsymbol{A}, \boldsymbol{B}$, Image of Dbx1;Ai148 slice preparation under Dodt contrast $(\boldsymbol{A})$ and fluorescence microscopy $(\boldsymbol{B})$. preBötC (red box) colocalized with the NAsc and the principle loop of the inferior olive $\left(\mathrm{IO}_{\text {loop }}\right)$. Scale bar for both $\boldsymbol{A}, \boldsymbol{B}: 500 \mu \mathrm{m}$. C, Voltage trajectory of a Dbx1 preBötC neuron during formation of a gramicidin perforated patch, firing in sync with XII nerve activity. Dodt image shows the patch pipette on the neuronal soma. The magenta arrow (labeled p.) points to the patch pipette, the cyan arrow (labeled soma) indicates the patched cell body. GCaMP6f image shows the neuron was $D b x 1$ derived. The lack of fluorescence in the Alexa Fluor 568 image verifies the integrity of the perforated patch. $\boldsymbol{D}$, Membrane voltage trajectory of the same neuron during blockade of chloride-mediated synaptic inhibition via addition of $5 \mu \mathrm{m}$ strychnine and $5 \mu \mathrm{m}$ picrotoxin in the bath. Alexa Fluor 568 continues to verify the integrity of the perforated patch. $\boldsymbol{E}$, The same neuron in a whole-cell patch configuration shows Alexa Fluor 568 enters the soma from the patch pipette. The Dbx 1 neuron is still rhythmically active and fires in sync with XII motor output. $\boldsymbol{F}$, Average integrated burst area during either control conditions (ctrl, blue) or during glycine and $\mathrm{GABA}_{\mathrm{A}}$ receptor blockade (disinhib, red). $\boldsymbol{G}$, Determining $\mathrm{E}_{\mathrm{CI}}$. Voltage trajectory before and after puffer application of $150 \mu \mathrm{m}$ glycine and $30 \mu \mathrm{m}$ muscimol in TTX. The average baseline membrane potential before puffer application was calculated from the previous $2 \mathrm{~s}$ of recording (orange box). The puff-evoked change in membrane voltage $\left(\Delta \mathrm{V}_{\mathrm{m}}\right)$ was the difference between baseline and the peak response. $\boldsymbol{H}$, Membrane potential changes in response to glycine and muscimol puffs plotted versus holding potential from a single representative experiment (same cell as $G$ ). $\mathrm{E}_{\mathrm{Cl}}$ (read from the $x$-axis coordinate where $\Delta \mathrm{V}_{\mathrm{m}}=0$ on the $y$-axis) is determined from linear regression (black line). $I, E_{C I}$ from 11 neurons (four Dbx1 neurons from four Dbx1;Ai148 mouse slices, seven wild-type neurons) plotted as a function of postnatal age. Line at $-48 \mathrm{mV}$ indicates the average $\mathrm{E}_{\mathrm{Cl}}$ across all neurons. 
perforated patch configuration to whole-cell and recorded the dialysis of Alexa Fluor 568 into the soma, concomitant with increased action potential amplitude, which confirmed the patch had not been breached during control and disinhibition data acquisition bouts (Fig. $6 D, E$ ).

Next, we measured $\mathrm{E}_{\mathrm{Cl}}$ in 11 preBötC neurons ( $n=7$ unspecified preBötC neurons from wild-type slices, and $n=4$ rhythmically active Dbx1 preBötC neurons, all from different slices). In the presence of TTX, pressure-pulse ejections of muscimol and glycine transiently perturbed the membrane potential, which reversed at or below $-45 \mathrm{mV}$ (Fig. 6G-l). $\mathrm{E}_{\mathrm{Cl}}$ measured $-48 \pm 6 \mathrm{mV}$ (mean \pm $\mathrm{SD}, n=11$ preBötC neurons recorded in $n=10$ slices). We observed no relationship between $\mathrm{E}_{\mathrm{Cl}}$ and age (Fig. 6/), which indicates that $\mathrm{E}_{\mathrm{Cl}}$ remains constant during $\mathrm{PO}-\mathrm{P} 3$.

\section{Discussion}

Eupnea and sigh rhythms are coordinated. The conventional understanding is that sigh breaths build off the crest of eupneic breaths, and then eupneic breaths resume after the post-sigh apnea, a pronounced delay that exceeds the gap between eupneic breaths normally. Here, we show that the longstanding conceptual framework for the eupnea-sigh relationship is oversimplified. We report previously unrecognized variability in the timing between eupneic and sigh breaths in vivo. The temporal coupling between eupnea and sigh rhythms, including its variability, may be attributable to dynamics within the preBötC because these same dynamics are mimicked in slice preparations that isolate the preBötC yet maintain both inspiratory and sigh rhythms.

The variability in the coordination of the inspiratory and sigh rhythms suggests that their coupling is weaker, relatively speaking, than previously appreciated. Sighs and sigh bursts in vitro with inspiratory-sigh intervals $\leq 0 \mathrm{~s}$ (where the sigh burst appeared coincident with, or before, the inspiratory burst) further reinforce this notion of flexibility in the relationship between a sigh burst and an associated inspiratory burst by showing that the inspiratory burst is not strictly necessary to trigger a sigh burst. Thus, a sigh can manifest independently, however, more often than not an inspiratory burst does trigger the sigh burst.

Could a sigh (or sigh burst in vitro) followed by a breath (or inspiratory burst in vitro) reflect a stand-alone sigh accompanied by a spurious artifact on the downslope of the sigh? Unlikely, we argue. In the case of animal movement during plethysmography, mice tend to move in bouts of activity lasting 1-10 s, which perturbs breathing over many consecutive cycles, and they rarely move in isolated 50 - to $100-\mathrm{ms}$ episodes that correspond to the duration of a putative eupneic breath whose peak follows a sigh (Fig. 2A). For preBötC field recordings, noise-driven network activity fluctuations with magnitudes large enough to be confused with inspiratory bursts are rare. Further, in vivo and in vitro, the observed breaths (bursts) that follow the sigh events match the preceding tidal volume (inspiratory burst amplitude), which argues that they are bona fide eupneic (inspiratory) events rather than recording artifacts. Nevertheless, if we accept the possibility that the trailing event is spurious, then those negative eupnea- sigh (or inspiratory-sigh) intervals would instead be closer to one, because the preceding eupneic or inspiratory event would have occurred one entire cycle away. This abnormally long eupnea-sigh (or inspiratory-sigh) interval would further support our conclusion that sighs follow eupneic or inspiratory events with variable intervals, and that a sigh burst has no obligatory link to the preceding inspiratory burst.

Environmental conditions like hypoxia (Janssen et al., 2000) or stress (Ramirez, 2014) modulate sigh frequency. Bombesin-like peptides delivered by parafacial inputs to the preBötC influence sigh frequency (Li et al., 2016). However, it is not clear whether modulation of sigh frequency would influence the eupnea-sigh coupling.

Sighs are $2-5 \times$ the tidal volume of eupneic breaths in vivo, whereas sigh bursts in vitro exceed inspiratory bursts by only 1 - to 2 -fold. Despite this disparity in sigh event magnitudes, the temporal relationship between the two rhythms measured in vivo or in vitro remains the same, as shown in the histograms from Figures 2, $3 A$. Those observations suggest that sigh magnitude does not influence eupnea-sigh (inspiratory-sigh) coupling. It is also remarkable that the sigh frequency remains constant (0.3-0.5 $\left.\mathrm{min}^{-1}\right)$ and the eupnea-sigh coupling pattern is conserved from intact adult mice to slices despite the fact that slices isolate the preBötC from all sensory feedback and some pattern-forming microcircuits. The robustness of the sigh frequency and the eupnea-sigh coupling suggests the sigh timing, and its coupling with eupnea, is largely attributable to microcircuit mechanisms within the preBötC.

We examined the mechanisms of coupling in vitro. Chloride-mediated synaptic inhibition is not responsible for the temporal coupling between inspiratory and sigh bursts. Rather, removing inhibition shortened the time between a sigh burst and the preceding inspiratory burst. This conclusion contradicts prior studies showing that blockade of glycinergic transmission decoupled sighs from their preceding inspiratory bursts and created freerunning sigh burst rhythms that are independent from ongoing inspiratory rhythms (Lieske et al., 2000; Chapuis et al., 2014; Toporikova et al., 2015).

We propose that the discrepancy between those prior results and our present findings are attributable to the late embryonic reversal of the chloride electrochemical gradient. Before embryonic day (E)15.5 in mice, the dominant expression of cotransporter NKCC1 in brainstem and spinal cord neurons elevates intracellular chloride concentration (Ren and Greer, 2006; Delpy et al., 2008; Viemari et al., 2011) such that chloride currents are inward (i.e., excitatory) at the baseline membrane potential of rhythmically active preBötC interneurons. Perinatally NKCC1 expression decreases in parallel with increasing expression of the chloride symporter, KCC2, which lowers intracellular chloride concentration (Stil et al., 2009; Gackière and Vinay, 2015). In the mature state, dominant KCC2 expression ensures that the chloride equilibrium potential is more hyperpolarized than spike threshold as well as baseline membrane potential during rhythmic activity; chloride currents are outward and inhibitory. 


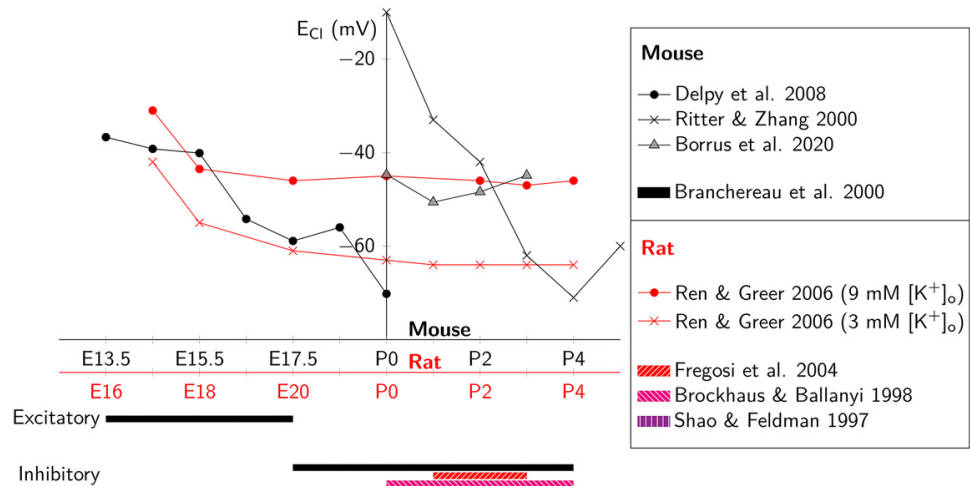

Figure 7. Meta-analysis of $\mathrm{E}_{\mathrm{Cl}}$ during development in hindbrain and spinal cord of mice and rats. Red reflects rat data, black reflects mouse. The abscissa shows age (in days) centered at birth (P0). The ordinate shows $\mathrm{E}_{\mathrm{Cl}}$ (in $\mathrm{mV}$ ) as a function of age. Below the $x$-axis, we show binary classification of $\mathrm{E}_{\mathrm{Cl}}$ as either above (excitatory) or below (inhibitory) baseline membrane potential as a function of developmental age. Our present data are labeled Borrus et al. (2020). We obtained ECl data from perinatal mice from: Branchereau et al. (2000), Delpy et al., 2008, Ritter and Zhang (2000), and data from the present manuscript, i.e., Borrus et al. (2020). We obtained ECl data from perinatal rats from: Brockhaus and Ballanyi (1998), Fregosi et al. (2004), Ren and Greer, 2006, as well as Shao and Feldman (1997).

Table 1: Summary of statistics from figures

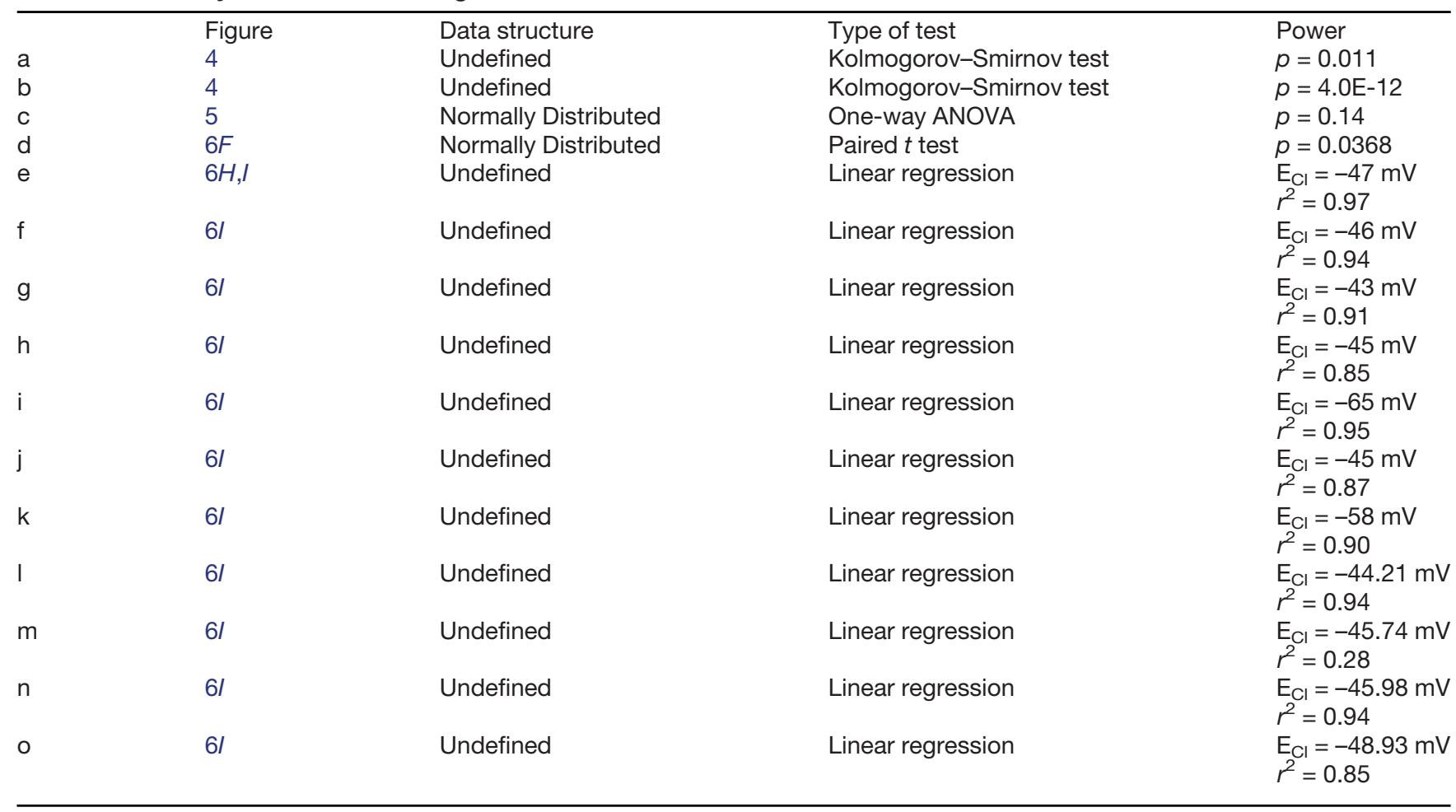

Here, we show, in early postnatal mouse development (P0-P4) with elevated (9 mM) $\left[\mathrm{K}^{+}\right]_{0}$ aCSF to boost slice excitability, that blocking chloride-mediated synapses leads to a larger depolarizing drive during inspiratory bursts. This suggests that $\mathrm{GABA}_{\mathrm{A}}$ and glycinergic inputs are effectively inhibitory, diminishing the effects of excitatory synaptic drive during inspiration. $\mathrm{E}_{\mathrm{Cl}}$ measured $-48 \mathrm{mV}$ during early postnatal development in the preBötC. This equilibrium potential is below spike threshold and approximates the level of baseline membrane potential during the interburst interval. Therefore,
$\mathrm{GABA}_{A}$ and glycinergic inputs either shunt the membrane, rendering it less responsive to excitatory (depolarizing) drive, or hyperpolarize it directly during the preinspiratory phase or during the inspiratory burst itself when the membrane potential trajectory exceeds $-48 \mathrm{mV}$. Thus, in our experimental context strychnine and picrotoxin truly block synaptic inhibition.

We meta-analyzed the development of $\mathrm{E}_{\mathrm{Cl}}$ in rodents in the context of our own $\mathrm{E}_{\mathrm{Cl}}$ data (Fig. 7). Most studies agree that $\mathrm{E}_{\mathrm{Cl}}$ switches from above to below baseline membrane potential (i.e., from excitatory to inhibitory) 
around birth, which is consistent with our measurement of $\mathrm{E}_{\mathrm{Cl}}=-48 \mathrm{mV}$. However, there is substantial variability, which may be attributable to rodent species and strain differences, as well as choice of $\left[\mathrm{K}^{+}\right]_{\mathrm{o}}$ in the aCSF, which impacts the electrochemical forces that operate in transporters.

Whereas we studied postnatal (P0-P4) mice exclusively, Chapuis et al. (2014) and Toporikova et al. (2015) studied embryonic mice (E15.5-E18.5). In embryonic mouse slices bathed in elevated $(8 \mathrm{~mm})\left[\mathrm{K}^{+}\right]_{\mathrm{o}} \mathrm{aCSF}, \mathrm{E}_{\mathrm{Cl}}$ is more likely to be above spike threshold and consequently glycinergic synapses serve to depolarize and evoke action potentials in preBötC neurons (Ren and Greer, 2006; Delpy et al., 2008). Under these conditions, we infer that glutamatergic, glycinergic, and GABAergic synapses are all effectively excitatory and link sigh bursts to their preceding inspiratory bursts. When net excitatory drive is perturbed (such as by blocking chloride-mediated synapses) then inspiratory-sigh coupling weakens, and the sigh rhythm proceeds in a manner that is temporally independent of the inspiratory rhythm. Those results, in conjunction with our work showing disinhibition strengthens inspiratory-sigh coupling, leads us to our primary conclusion: that excitatory (not inhibitory) synaptic transmission links the inspiratory and sigh rhythms of the preBötC.

Development and chloride gradients might also explain the discrepancies between our results and Lieske et al. (2000), who also concluded that glycinergic synapses couple inspiratory and sigh rhythms. Those authors reported using mice aged zero to two weeks, a postnatal window that overlaps and extends beyond ours. We surmise that their sigh burst experiments were performed exclusively or predominantly using preparations from PO mice with immature chloride gradients. As Figure 7 shows, Ritter and Zhang (2000) employed perforatedpatch recordings and reported that $\mathrm{E}_{\mathrm{Cl}}$ remained depolarizing and ostensibly excitatory as late as P3. Therefore, it is conceivable, even likely, that the same explanation holds for Lieske et al. (2000). Namely, chloride gradients favoring inward currents (with suprathreshold reversal potential) render glycine synapses ostensibly excitatory.

Chloride-mediated synaptic inhibition does not contribute to the post-sigh apnea. Instead, we suggest the postsigh apnea is likely caused by activation of the intrinsic cellular mechanisms that help terminate inspiratory bursts, which are recruited to a greater degree during sigh events (compared with typical inspiratory cycles). These burst-terminating mechanisms include activity-dependent outward currents such as the electrogenic $\mathrm{Na} / \mathrm{K}$ ATPase pump current, $\mathrm{Na}^{+}$-dependent $\mathrm{K}^{+}$current, and ATP-dependent $\mathrm{K}^{+}$current (Del Negro et al., 2009; Krey et al., 2010), as well as excitatory synaptic depression (Kottick and Del Negro, 2015; Guerrier et al., 2015). The magnitude of inspiratory burst-related depolarization directly evokes corresponding levels of post-burst hyperpolarization in preBötC neurons, from which the neurons must recover before generating the next inspiratory burst (Baertsch et al., 2018). The sigh burst in this context is an extreme version of that same mechanism: the increased magnitude and duration of the sigh event correspondingly evokes larger-than-average activity-dependent refractory (outward) currents and depresses excitatory synapses to a greater extent than during typical inspiratory bursts of lower magnitude and duration. The larger-than-average hyperpolarization (and depressed synapses) extends the duration of the interburst interval, thus creating the postsigh apnea.

Here, we demonstrate that chloride-mediated synaptic inhibition plays no obligatory role in coupling the inspiratory and sigh rhythms in P0-P4 mice in vitro. Although sigh bursts are often closely preceded by inspiratory bursts, their temporal coordination is more variable than previously documented. We speculate that this principle may also apply to juvenile and adult stages because $\mathrm{E}_{\mathrm{Cl}}$ is expected to remain below spike threshold and inhibitory; in fact, we expect it to descend lower than $-48 \mathrm{mV}$ during further maturation. A model of the preBötC core that generates inspiratory and sigh oscillations should emphasize the primacy of excitatory synaptic interactions, which probably extends to embryonic development when chloride-mediated synapses are functionally excitatory.

\section{References}

Ashhad S, Feldman JL (2020) Emergent elements of inspiratory rhythmogenesis: network synchronization and synchrony propagation. Neuron 106:482-497.e4.

Baertsch NA, Baertsch HC, Ramirez JM (2018) The interdependence of excitation and inhibition for the control of dynamic breathing rhythms. Nat Commun 9.1:843.

Barry PH, Lynch JW (1991) Liquid junction potentials and small cell effects in patch-clamp analysis. J Membr Biol 121:101-117.

Bielle F, Griveau A, Narboux-Nême N, Vigneau S, Sigrist M, Arber S, Wassef M, Pierani A (2005) Multiple origins of Cajal-Retzius cells at the borders of the developing pallium. Nat Neurosci 8:1002-1012.

Bouvier J, Thoby-Brisson M, Renier N, Dubreuil V, Ericson J, Champagnat J, Pierani A, Chédotal A, Fortin G (2010) Hindbrain interneurons and axon guidance signaling critical for breathing. Nat Neurosci 13:1066-1074

Branchereau P, Morin D, Bonnot A, Ballion B, Chapron J, Viala D (2000) Development of lumbar rhythmic networks: from embryonic to neonate locomotor-like patterns in the mouse. Brain Res Bull 53:711-718.

Brockhaus J, Ballanyi K (1998) Synaptic inhibition in the isolated respiratory network of neonatal rats. Eur J Neurosci 10:3823-3839.

Chapuis C, Autran S, Fortin G, Simmers J, Thoby-Brisson M (2014) Emergence of sigh rhythmogenesis in the embryonic mouse: sigh in mouse embryo. J Physiol 592:2169-2181.

Cherniack NS, von Euler C, Głogowska M, Homma I (1981) Characteristics and rate of occurrence of spontaneous and provoked augmented breaths. Acta Physiol Scand 111:349-360.

Cui Y, Kam K, Sherman D, Janczewski WA, Zheng Y, Feldman JL (2016) Defining preBötzinger complex rhythm- and pattern-generating neural microcircuits in vivo. Neuron 91:602-614.

Daigle TL, Madisen L, Hage TA, Valley MT, Knoblich U, Larsen RS, Takeno MM, Huang L, Gu H, Larsen R, Mills M, Bosma-Moody A, Siverts LA, Walker M, Graybuck LT, Yao Z, Fong O, Nguyen TN, Garren E, et al. (2018) A suite of transgenic driver and reporter mouse lines with enhanced brain-cell-type targeting and functionality. Cell 174:465-480.e22.

Del Negro CA, Morgado-Valle C, Feldman JL (2002) Respiratory rhythm: an emergent network property? Neuron 34:821-830.

Del Negro CA, Kam K, Hayes JA, Feldman JL (2009) Asymmetric control of inspiratory and expiratory phases by excitability in the respiratory network of neonatal mice in vitro: asymmetric control of respiratory phases. J Physiol 587:1217-1231. 
Del Negro CA, Funk GD, Feldman JL (2018) Breathing matters. Nat Rev Neurosci 19:351-367.

Delpy A, Allain A-E, Meyrand P, Branchereau P (2008) NKCC1 cotransporter inactivation underlies embryonic development of chloride-mediated inhibition in mouse spinal motoneuron. J Physiol 586:1059-1075.

Feldman JL, Kam K (2015) Facing the challenge of mammalian neural microcircuits: taking a few breaths may help. J Physiol 593:3-23.

Fregosi RF, Luo Z, lizuka M (2004) $\mathrm{GABA}_{A}$ receptors mediate postnatal depression of respiratory frequency by barbiturates. Respir Physiol Neurobiol 140:219-230.

Funk GD, Greer JJ (2013) The rhythmic, transverse medullary slice preparation in respiratory neurobiology: contributions and caveats. Respir Physiol Neurobiol 186:236-253.

Funk GD, Smith JC, Feldman JL (1993) Generation and transmission of respiratory oscillations in medullary slices: role of excitatory amino acids. J Neurophysiol 70:1497-1515.

Gackière F, Vinay L (2015) Contribution of the potassium-chloride cotransporter KCC2 to the strength of inhibition in the neonatal rodent spinal cord in vitro. J Neurosci 35:5307-5316.

Gray PA, Hayes JA, Ling GY, Llona I, Tupal S, Picardo MCD, Ross SE, Hirata T, Corbin JG, Eugenín J, Del Negro CA (2010) Developmental origin of preBötzinger complex respiratory neurons. J Neurosci 30:14883-14895.

Guerrier C, Hayes JA, Fortin G, Holcman D (2015) Robust network oscillations during mammalian respiratory rhythm generation driven by synaptic dynamics. Proc Natl Acad Sci USA 112:97289733.

Hirata T, Li P, Lanuza GM, Cocas LA, Huntsman MM, Corbin JG (2009) Identification of distinct telencephalic progenitor pools for neuronal diversity in the amygdala. Nat Neurosci 12:141-149.

Janssen PL, Williams JS, Fregosi RF (2000) Consequences of periodic augmented breaths on tongue muscle activities in hypoxic rats. J Appl Physiol 88:1915-1923.

Kottick A, Del Negro CA (2015) Synaptic depression influences inspiratory-expiratory phase transition in Dbx1 interneurons of the preBötzinger complex in neonatal mice. J Neurosci 35:1160611611.

Kottick A, Martin CA, Del Negro CA (2017) Fate mapping neurons and glia derived from Dbx1-expressing progenitors in mouse preBötzinger complex. Physiol Rep 5:e13300.

Krey RA, Goodreau AM, Arnold TB, Del Negro CA (2010) Outward currents contributing to inspiratory burst termination in preBötzinger complex neurons of neonatal mice studied in vitro. Front Neural Circuits 4:124.

Li P, Yackle K (2017) Sighing. Curr Biol 27:R88-R89.

Li P, Janczewski WA, Yackle K, Kam K, Pagliardini S, Krasnow MA, Feldman JL (2016) The peptidergic control circuit for sighing. Nature 530:293-297.

Lieske SP, Ramirez JM (2006a) Pattern-specific synaptic mechanisms in a multifunctional network. I. Effects of alterations in synapse strength. J Neurophysiol 95:1323-1333.

Lieske SP, Ramirez JM (2006b) Pattern-specific synaptic mechanisms in a multifunctional network. II. Intrinsic modulation by metabotropic glutamate receptors. J Neurophysiol 95:1334-1344.

Lieske SP, Thoby-Brisson M, Telgkamp P, Ramirez JM (2000) Reconfiguration of the neural network controlling multiple breathing patterns: eupnea, sighs and gasps. Nat Neurosci 3:600-607.

National Research Council (U.S.) (2011) Guide for the care and use of laboratory animals, Ed 8. Washington, DC: National Academies Press.

Neher E (1992) Correction for liquid junction potentials in patch clamp experiments. Methods Enzymol 207:123-131.

Orem J, Trotter RH (1993) Medullary respiratory neuronal activity during augmented breaths in intact unanesthetized cats. J Appl Physiol 74:761-769.
Picardo MCD, Weragalaarachchi KTH, Akins VT, Del Negro CA (2013) Physiological and morphological properties of Dbx1-derived respiratory neurons in the pre-Botzinger complex of neonatal mice. J Physiol 591:2687-2703.

Ramirez JM (2014) The integrative role of the sigh in psychology, physiology, pathology, and neurobiology. Prog Brain Res 209:91129.

Rekling JC, Shao XM, Feldman JL (2000) Electrical coupling and excitatory synaptic transmission between rhythmogenic respiratory neurons in the preBötzinger complex. J Neurosci 20:RC113.

Ren J, Greer J (2006) Modulation of respiratory rhythmogenesis by chloride-mediated conductances during the perinatal period. $\mathrm{J}$ Neurosci 26:3721-3730.

Ritter B, Zhang W (2000) Early postnatal maturation of GABA $A_{A}$-mediated inhibition in the brainstem respiratory rhythm-generating network of the mouse. Eur J Neurosci 12:2975-2984.

Ruangkittisakul A, Schwarzacher SW, Secchia L, Ma Y, Bobocea N, Poon BY, Funk GD, Ballanyi K (2008) Generation of eupnea and sighs by a spatiochemically organized inspiratory network. J Neurosci 28:2447-2458.

Ruangkittisakul A, Panaitescu B, Ballanyi $\mathrm{K}(2011) \mathrm{K}(+)$ and $\mathrm{Ca}^{2}(+)$ dependence of inspiratory-related rhythm in novel "calibrated" mouse brainstem slices. Respir Physiol Neurobiol 175:37-48.

Ruangkittisakul A, Kottick A, Picardo MCD, Ballanyi K, Del Negro CA (2014) Identification of the pre-Bötzinger complex inspiratory center in calibrated "sandwich" slices from newborn mice with fluorescent Dbx1 interneurons. Physiol Rep 2:e12111.

Shao XM, Feldman JL (1997) Respiratory rhythm generation and synaptic inhibition of expiratory neurons in pre-Bötzinger complex: differential roles of glycinergic and GABAergic neural transmission. J Neurophysiol 77:1853-1860.

Smith JC, Ellenberger H, Ballanyi K, Richter D, Feldman J (1991) PreBotzinger complex: a brainstem region that may generate respiratory rhythm in mammals. J Gen Physiol 83:633-656.

Stil A, Liabeuf S, Jean-Xavier C, Brocard C, Viemari J-C, Vinay L (2009) Developmental up-regulation of the potassium-chloride cotransporter type 2 in the rat lumbar spinal cord. Neuroscience 164:809-821.

Toporikova N, Chevalier M, Thoby-Brisson M (2015) Sigh and eupnea rhythmogenesis involve distinct interconnected subpopulations: a combined computational and experimental study. eNeuro 2:ENEURO.0074-14.2015.

Tryba AK, Peña F, Lieske SP, Viemari JC, Thoby-Brisson M, Ramirez JM (2008) Differential modulation of neural network and pacemaker activity underlying eupnea and sigh-breathing activities. J Neurophysiol 99:2114-2125.

Vann NC, Pham FD, Hayes JA, Kottick A, Del Negro CA (2016) Transient suppression of $\mathrm{Dbx} 1$ preBötzinger interneurons disrupts breathing in adult mice. PLoS One 11:e0162418.

Vann NC, Pham FD, Dorst KE, Del Negro CA (2018) Dbx1 preBötzinger complex interneurons comprise the core inspiratory oscillator for breathing in unanesthetized adult mice. eNeuro 5: ENEURO.0130-18.2018.

Viemari JC, Bos R, Boulenguez P, Brocard C, Brocard F, Bras H, Coulon P, Liabeuf S, Pearlstein E, Sadlaoud K, Stil A, Tazerart S, Vinay L (2011) Importance of chloride homeostasis in the operation of rhythmic motor networks. Prog Brain Res 188:3-14.

Wallén-Mackenzie A, Gezelius H, Thoby-Brisson M, Nygård A, Enjin A, Fujiyama F, Fortin G, Kullander K (2006) Vesicular glutamate transporter 2 is required for central respiratory rhythm generation but not for locomotor central pattern generation. J Neurosci 26:12294-12307.

Wang X, Hayes JA, Revill AL, Song H, Kottick A, Vann NC, LaMar MD, Picardo MCD, Akins VT, Funk GD, Del Negro CA (2014) Laser ablation of Dbx1 neurons in the pre-Bötzinger complex stops inspiratory rhythm and impairs output in neonatal mice. Elife 3 : e03427. 